\title{
ON APPLICATIONS OF DIFFERENTIAL SUBORDINATION AND SUPERORDINATION
}

\author{
N. MARIKKANNAN AND C. GANESAMOORTHY
}

\begin{abstract}
In the present investigation we obtain the sufficient conditions for normalized analytic functions $f$ to satisfy

$$
q_{1}<\frac{f^{2}}{z^{2} f^{\prime}}<q_{2},
$$

where $q_{1}$ and $q_{2}$ are univalent functions with $q_{1}(0)=q_{2}(0)=1$. Also we obtain the sandwich results involving Carlson-Shaffer linear operator, Sălăgean derivative and Ruscheweyh derivative.
\end{abstract}

\section{Introduction}

Let $\mathscr{A}$ be the class of normalized analytic functions $f$ in the open unit disk $\Delta:=\{z \in \mathbb{C}$ : $|z|<1$ s satisfying $f(0)=f^{\prime}(0)-1=0$. For two functions $f$ and $g$ given by

$$
f(z):=z+\sum_{n=2}^{\infty} f_{n} z^{n} \text { and } g(z):=z+\sum_{n=2}^{\infty} g_{n} z^{n},
$$

their Hadamard product or convolution is defined as

$$
(f * g)(z):=z+\sum_{n=2}^{\infty} f_{n} g_{n} z^{n} .
$$

Define the function $\varphi(a, c ; z)$ by

$$
\varphi(a, c ; z):=\sum_{n=0}^{\infty} \frac{(a)_{n}}{(c)_{n}} z^{n+1} \quad(c \neq 0,-1,-2, \ldots ; z \in \Delta),
$$

where $(\lambda)_{n}$ is the Pocchhammer symbol defined by

$$
(\lambda)_{n}:= \begin{cases}1 & (n=0) \\ \lambda(\lambda+1)(\lambda+2) \cdots(\lambda+n-1) & (n=1,2,3 \ldots) .\end{cases}
$$

Corresponding to the function $\varphi(a, c ; z)$ Carlson-Shaffer [5] introduced an operator $L(a, c)$ for $f \in \mathscr{A}$ using Hadamard product as follows:

$$
L(a, c) f(z):=\varphi(a, c ; z) * f(z)
$$

Received May 16, 2007; Revised November 5, 2007.

2000 Mathematics Subject Classification. Primary30C45, secondary 30C80.

Key words and phrases. Differential subordination, superordination, Carlson-Shaffer linear operator, Sălăgean derivative and Ruscheweyh derivative. 


$$
=z+\sum_{n=1}^{\infty} \frac{(a)_{n}}{(c)_{n}} a f_{n+1} z^{n+1} .
$$

Note that $L(a, a) f=f ; L(2,1) f=z f^{\prime}$ and $L(\delta+1,1) f=D^{\delta} f$, where $D^{\delta} f$ is the Ruscheweyh derivative of order $\delta[6]$.

Sălăgean derivative operator of order $m$ [7] for $f \in \mathscr{A}$, denoted by $\mathscr{D}^{m} f$, defined as

$$
\mathscr{D}^{m} f(z)=z+\sum_{n=2}^{\infty} n^{m} a_{n} z^{n} .
$$

Note that $\mathscr{D}^{0} f=f$ and $\mathscr{D}^{1} f=z f^{\prime}$.

Let $\mathscr{H}$ denotes the class of functions analytic in $\Delta$ and $\mathscr{H}[a, n]$ denotes the subclass of $\mathscr{H}$ consisting of functins of the form $f(z)=a+a_{n} z^{n}+a_{n+1} z^{n+1}+\cdots$. For two analytic functions $f, F \in \mathscr{H}$ we say $F$ is superordinate to $f$, if $f$ is subordinate to $F$. Let $p, h \in \mathscr{H}$ and let $\phi(r, s, t ; z): \mathbb{C}^{3} \times \Delta \rightarrow \mathbb{C}$. If $p$ and $\phi\left(p, z p^{\prime}, z^{2} p^{\prime \prime} ; z\right)$ are univalent and if $p$ satisfies the second order superordination

$$
h<\phi\left(p, z p^{\prime}, z^{2} p^{\prime \prime} ; z\right),
$$

then $p$ is the solution of the differential superordination (1.1). An analytic function $q$ is called subordinant, if $q<p$ for all $p$ satisfying (1.1). A univalent subordinant $\tilde{q}$ thatsatisfies $q<\tilde{q}$ for all subordinants $q$ of (1.1), is said to be best subordinant.Recently Miller and Mocanu [3] obtained conditionson $h, q$ and $\phi(r, s, t ; z)$ to satisfy the following:

$$
h<\phi\left(p, z p^{\prime}, z^{2} p^{\prime \prime} ; z\right) \Rightarrow q<p .
$$

Using the results of Miller and Mocanu [3], Bulboacă have considered certain classes of first order differential superordinations [2] as well as superordination preserving integral operators [1].

In the present investigation we give some application of first order differential subordination and superordination to obtain sufficient conditions for normalized analytic functions $f$ to satisfy

$$
q_{1} \prec \frac{f^{2}}{z^{2} f^{\prime}} \prec q_{2}
$$

where $q_{1}$ and $q_{2}$ are univalent in $\Delta$. Also applications to Carlson-Shaffer linear operator and Sălăgean derivative are studied.

\section{Preliminaries}

For the present investigation we need the following definition and results.

Definition 2.1. [3, Definition 2, p.817] Denote by $\mathscr{Q}$, the set of all functions $f$ that are analytic and univalent in $\bar{\Delta} \backslash E(f)$, where

$$
E(f):=\left\{\zeta \in \partial \Delta: \lim _{z \rightarrow \zeta} f(z)=\infty\right\}
$$


and are such that $f^{\prime}(\zeta) \neq 0$ for $\zeta \in \partial \Delta \backslash E(f)$.

Theorem 2.1. (cf. Miller and Mocanu [4, Theorem 3.4h, p.132]) Let $q$ be univalent in $\Delta$ and $\theta$ and $\phi$ be analytic in a domain D containing $q(\Delta)$ with $\phi(w) \neq 0$, when $w \in q(\Delta)$. Set $Q=z q^{\prime} \phi(q), h=\theta(q)+Q$. Suppose that

(i) $Q$ is starlike univalent in $\Delta$ and

(ii) $\Re\left\{\frac{z h^{\prime}}{Q}\right\}>0$ for $z \in \Delta$.

If $p$ is analytic in $\Delta$ with $p(\Delta) \subseteq D$ and

$$
\theta(p)+z p^{\prime} \phi(p)<\theta(q)+z q^{\prime} \phi(q)
$$

then

$$
p<q
$$

and $q$ is the best dominant.

Theorem 2.2. [2] Let $q$ be univalent in $\Delta$ and $\theta$ and $\phi$ be analytic in domain $D$ containing $q(\Delta)$. Suppose that

(i) $\Re\left(\frac{\theta^{\prime}(q)}{\phi(q)}\right) \geq 0$ for $z \in \Delta$ and

(ii) $g=z q^{\prime} \phi(q)$ is starlike univalent in $\Delta$.

If $p \in \mathscr{H}[q(0), 1] \cap \mathscr{Q}$ with $p(\Delta) \subseteq D$ and $\theta(p)+z p^{\prime} \phi(p)$ is univalent in $\Delta$, and

$$
\theta(q)+z q^{\prime} \phi(q)<\theta(p)+z p^{\prime} \phi(p),
$$

then

$$
q<p
$$

and $q$ is the best subordinant.

\section{Application to Analytic Functions}

Theorem 3.1. Let $0 \neq \alpha \in \mathbb{C}$ and $\Re\left\{\frac{1}{\alpha}\right\}>0$. Let $q$ be convex univalent in $\Delta$ with $q(0)=1$. Let

$$
\psi_{1}:=\frac{2 \alpha f}{z}+\frac{f^{2}}{z^{2} f^{\prime}}\left[(1-2 \alpha)-\frac{\alpha z f^{\prime \prime}}{f^{\prime}}\right]
$$

and $\chi_{1}:=q+\alpha z q^{\prime}$. Let $f \in \mathscr{A}$, and $\frac{f^{2}}{z^{2} f^{\prime}} \in \mathscr{H}[1,1] \cap \mathscr{Q}$ and $\psi_{1}$ is univalent in $\Delta$.

(i) If $\psi_{1}<\chi_{1}$ then

$$
\frac{f^{2}}{z^{2} f^{\prime}}<q
$$

where $q$ is the best dominant. 
(ii) If $\chi_{1}<\psi_{1}$ then

$$
q<\frac{f^{2}}{z^{2} f^{\prime}}
$$

where $q$ is the best subordinant.

Proof. Define the function $p$ by

$$
p:=\frac{f^{2}}{z^{2} f^{\prime}}
$$

A computation using (3.2) shows that

$$
\frac{z p^{\prime}}{p}=\frac{2 z f^{\prime}}{f}-\frac{z f^{\prime \prime}}{f^{\prime}}-2
$$

Also we note that an application of (3.3) yields

$$
\begin{aligned}
\psi_{1} & =\frac{2 \alpha f}{z}+\frac{f^{2}}{z^{2} f^{\prime}}\left[(1-2 \alpha)-\frac{\alpha z f^{\prime \prime}}{f^{\prime}}\right] \\
& =p+\alpha z p^{\prime},
\end{aligned}
$$

and this can be written as (2.1) when $\theta(w)=w$ and $\phi(w)=\alpha$. Note that $\phi(w) \neq 0$ and $\theta$ and $\phi$ are analytic in $\mathbb{C}$. Set

$$
\begin{aligned}
Q & :=\alpha z q^{\prime}, \\
h & :=\theta(q)+Q \\
& =q+\alpha z q^{\prime} .
\end{aligned}
$$

In light of the hypothesis of Theorem 2.1, we see that $Q$ is starlike and

$$
\Re\left\{\frac{z h^{\prime}}{Q}\right\}=\Re\left\{\frac{1}{\alpha}+\left(1+\frac{z q^{\prime \prime}}{q^{\prime}}\right)\right\}>0 .
$$

By an application of Theorem 2.1 we conclude that $p<q$ or

$$
\frac{f^{2}}{z^{2} f^{\prime}}<q
$$

Note that

$$
\Re\left\{\frac{\theta^{\prime}(q)}{\phi(q)}\right\}=\Re\left\{\frac{1}{\alpha}\right\} \geq 0 .
$$

Hence the result (ii) of Theorem 3.1 follows as a similar application of Theorem 2.2.

By making use of Theorem 3.1 we get the following sandwich type result.

Theorem 3.2. Let $0 \neq \alpha \in \mathbb{C}$ and $\Re\left\{\frac{1}{\alpha}\right\}>0$. Let $q_{i}$ for $i=1,2$ be convex univalent in $\Delta$, with $q_{i}(0)=1$. Let $\chi_{i}=q_{i}+\alpha z q_{i}^{\prime}$ for $i=1,2$ and $\psi_{1}$ as given by (3.1) be univalent in $\Delta$. If $f \in \mathscr{A}, \frac{f^{2}}{z^{2} f^{\prime}} \in \mathscr{H}[1,1] \cap \mathscr{Q}$ and

$$
\chi_{1}<\psi_{1}<\chi_{2}
$$


then

$$
q_{1} \prec \frac{f^{2}}{z^{2} f^{\prime}} \prec q_{2}
$$

where $q_{1}$ and $q_{2}$ are respectively the best subordinant and best dominant.

Theorem 3.3. Let $\alpha, \beta$ and $\gamma$ be complex numbers and $\gamma \neq 0$. Let $q$ be a convex univalent functions in $\Delta$ with $q(0)=1$ and $\frac{\gamma z q^{\prime}}{q}$ is starlike univalent in $\Delta$. Let

$$
\psi_{2}:=(\alpha-2 \gamma)+\frac{2 \gamma z f^{\prime}}{f}+\frac{\beta f^{2}}{z^{2} f^{\prime}}-\frac{\gamma z f^{\prime \prime}}{f^{\prime}}
$$

and $\chi_{2}=\alpha+\beta q+\frac{\gamma z q^{\prime}}{q}$. Let $f \in \mathscr{A}$ and $\frac{f^{2}}{z^{2} f^{\prime}} \in \mathscr{H}[1,1] \cap \mathscr{Q}$ and $\psi_{2}$ is univalent in $\Delta$.

(i) If $q$ satisfies

$$
\Re\left\{\frac{\beta q}{\gamma}-\frac{z q^{\prime}}{q}\right\}>0
$$

then

$$
\psi_{2} \prec \chi_{2} \Rightarrow \frac{f^{2}}{z^{2} f^{\prime}} \prec q
$$

where $q$ is the best dominant.

(ii) If $q$ satisfies

$$
\Re\left\{\frac{\beta q}{\gamma}\right\}>0
$$

then

$$
\chi_{2} \prec \psi_{2} \Rightarrow q \prec \frac{f^{2}}{z^{2} f^{\prime}}
$$

where $q$ is the best subordinant.

Proof. Define the function $p$ by

$$
p:=\frac{f^{2}}{z^{2} f^{\prime}} .
$$

A simple computation using (3.3) shows that

$$
\begin{aligned}
\psi_{2} & :=(\alpha-2 \gamma)+\frac{2 \gamma z f^{\prime}}{f}+\frac{\beta f^{2}}{z^{2} f^{\prime}}-\frac{\gamma z f^{\prime \prime}}{f^{\prime}} \\
& =\alpha+\beta p+\frac{\gamma z p^{\prime}}{p}
\end{aligned}
$$

This can be written as (2.1) when $\theta(w):=\alpha+\beta w$ and $\phi(w):=\frac{\gamma}{w}$. Note that $\theta$ and $\phi$ are analytic in $\mathbb{C} \backslash\{0\}$. Set

$$
Q:=\frac{\gamma z q^{\prime}}{q}
$$




$$
\begin{aligned}
h & :=\alpha+\beta q+Q \\
& =\alpha+\beta q+\frac{\gamma z q^{\prime}}{q}
\end{aligned}
$$

In light of hypothesis of Theorem 2.1 we see that $Q$ is starlike and

$$
\Re\left\{\frac{z h^{\prime}}{Q)}\right\}=\Re\left\{\frac{\beta q}{\gamma}-\frac{z q^{\prime}}{q}+\left(1+\frac{z q^{\prime \prime}}{q}\right)\right\}>0 .
$$

By an application of Theorem 2.1 we conclude that

$$
\frac{f^{2}}{z^{2} f^{\prime}}<q
$$

The result (ii) of Theorem (3.3) follows as a similar exercise using Theorem 2.2.

\section{Application to Carlson-Shaffer Operator}

Theorem 4.1. Let $0 \neq \alpha \in \mathbb{C}$ and $\Re\left\{\frac{1}{\alpha} t\right\}>0$. Let $q$ be convex univalent in $\Delta$ with $q(0)=1$. Let

$$
\psi_{3}:=\frac{\{L(a, c) f\}^{2}}{z L(a+1, c) f}\left[1+\alpha(1-a)-\frac{\alpha(a+1) L(a+2, c) f}{L(a+1, c) f}\right]+\frac{2 a \alpha}{z} L(a, c) f
$$

and $\chi_{3}:=q+\alpha z q^{\prime}$. Let $f \in \mathscr{A}$ and $\frac{\{L(a, c) f\}^{2}}{z L(a+1, c) f} \in \mathscr{H}[1,1] \cap \mathscr{Q}$ and $\psi_{3}$ is univalent in $\Delta$.

(i) If $\psi_{3}<\chi_{3}$ then

$$
\frac{\{L(a, c) f\}^{2}}{z L(a+1, c) f}<q
$$

where $q$ is the best dominant.

(ii) If $\chi_{3}<\psi_{3}$ then

$$
q<\frac{\{L(a, c) f\}^{2}}{z L(a+1, c) f}
$$

where $q$ is the best subordinant.

Proof. Define the function $p$ by

$$
p:=\frac{\{L(a, c) f\}^{2}}{z L(a+1, c) f} .
$$

A simple computation using (4.1) gives

$$
\frac{z p^{\prime}}{p}=\frac{2 z(L(a, c) f)^{\prime}}{L(a, c) f}-1-\frac{z(L(a+1, c) f)^{\prime}}{L(a+1, c) f} .
$$

By using the identity

$$
z(L(a, c) f)^{\prime}=a L(a+1, c) f-(a-1) L(a, c) f
$$


in (4.2) we obtain

$$
\frac{z p^{\prime}}{p}=(1-a)+\frac{2 a L(a+1, c) f}{L(a, c) f}-(a+1) \frac{L(a+2, c) f}{L(a+1, c) f} .
$$

Note that

$$
\begin{aligned}
\psi_{3} & :=\frac{\{L(a, c) f\}^{2}}{z L(a+1, c) f}\left[1+\alpha(1-a)-\frac{\alpha(a+1) L(a+2, c) f}{L(a+1, c) f}\right]+\frac{2 a \alpha}{z} L(a, c) f \\
& =p+\alpha z p^{\prime}
\end{aligned}
$$

and this can be written as (2.1) when $\theta(w)=w$ and $\phi(w)=\alpha$. Hence the result (i) follows as an application of Theorem (2.1). The proof of result (ii) of Theorem 4.1 follows as a similar application of Theorem 2.2.

By taking $a=\delta+1$ and $c=1$ we get the following result involving Ruscheweyh derivative.

Corollary 4.2. Let $0 \neq \alpha \in \mathbb{C}$ and $\Re\left\{\frac{1}{\alpha}\right\}>0$. Let $q$ be convex univalent in $\Delta$ with $q(0)=1$. Let

$$
\psi:=\frac{\left\{D^{\delta} f\right\}^{2}}{z D^{\delta+1} f}\left[1+\alpha(1-a)-\alpha(a+1) \frac{D^{\delta+2} f}{D^{\delta+1} f}\right]+\frac{2 a \alpha}{z} D^{\delta} f .
$$

and $\chi:=q+\alpha z q^{\prime}$. Let $f \in \mathscr{A}$ and $\frac{\left\{D^{\delta} f\right\}^{2}}{z D^{\delta+1} f} \in \mathscr{H}[1,1] \cap \mathscr{Q}$ and $\psi$ is univalent in $\Delta$.

(i) If $\psi<\chi$ then

$$
\frac{\left\{D^{\delta} f\right\}^{2}}{z D^{\delta+1} f}<q
$$

where $q$ is the best dominant.

(ii) If $\chi<\psi$ then

$$
q<\frac{\left\{D^{\delta} f\right\}^{2}}{z D^{\delta+1} f}
$$

where $q$ is the best subordinant.

Theorem 4.3. Let $\alpha, \beta$ and $\gamma$ be complex numbers with $\gamma \neq 0$. Let $q$ be a convex univalent in $\Delta$ with $q(0)=1$ and $\frac{\gamma z q^{\prime}}{q}$ is starlike univalent in $\Delta$. Let

$$
\psi_{4}:=\alpha+\gamma(1-a)+\frac{\beta\{L(a, c) f\}^{2}}{z L(a+1, c) f}+\frac{2 a \gamma L(a+1, c) f}{L(a, c) f}-\frac{\gamma(a+1) L(a+2, c) f}{L(a+1, c) f}
$$

and $\chi_{4}:=\alpha+\beta q+\frac{\gamma z q^{\prime}}{q}$. Let $f \in \mathscr{A}$ and $\frac{\{L(a, c) f\}^{2}}{z L(a+1, c) f} \in \mathscr{H}[1,1] \cap \mathscr{Q}$ and $\psi_{4}$ is univalent in $\Delta$.

(i) If $q$ satisfies (3.4) then

$$
\psi_{4} \prec \chi_{4} \Rightarrow \frac{\{L(a, c) f\}^{2}}{z L(a+1, c) f}<q
$$

where $q$ is the best dominant. 
(ii) If $q$ satisfies (3.5) then

$$
\chi_{4} \prec \psi_{4} \Rightarrow q \prec \frac{\{L(a, c) f\}^{2}}{z L(a+1, c) f}
$$

where $q$ is the best subordinant.

Proof. The proof of the Theorem 4.3 is similar to that of Theorem 4.1, where $\theta(w)=\alpha+\beta w$ and $\phi(w)=\frac{\gamma}{w}$.

By taking $a=\delta+1$ and $c=1$ we get the following result involving Ruscheweyh derivative.

Corollary 4.4. Let $\alpha, \beta$ and $\gamma$ be complex numbers with $\gamma \neq 0$. Let $q$ be a convex univalent in $\Delta$ with $q(0)=1$ and $\frac{\gamma z q^{\prime}}{q}$ is starlike univalent in $\Delta$. Let

$$
\psi_{5}:=\alpha+\gamma(1-a)+\frac{\beta\left\{D^{\delta} f\right\}^{2}}{z D^{\delta+1} f}+\frac{2 a \gamma D^{\delta+1} f}{D^{\delta} f}-\frac{\gamma(a+1) D^{\delta+2} f}{D^{\delta+1} f}
$$

and $\chi_{5}:=\alpha+\beta q+\frac{\gamma z q^{\prime}}{q}$. Let $f \in \mathscr{A}$ and $\frac{\left\{D^{\delta} f\right\}^{2}}{z D^{\delta+1} f} \in \mathscr{H}[1,1] \cap \mathscr{Q}$ and $\psi_{5}$ is univalent in $\Delta$.

(i) If $q$ satisfies (3.4) then

$$
\psi_{5} \prec \chi_{5} \Rightarrow \frac{\left\{D^{\delta} f\right\}^{2}}{z D^{\delta+1} f} \prec q
$$

where $q$ is the best dominant.

(ii) If $q$ satisfies (3.5) then

$$
\chi_{5} \prec \psi_{5} \Rightarrow q \prec \frac{\left\{D^{\delta} f\right\}^{2}}{z D^{\delta+1} f}
$$

where $q$ is the best subordinant.

\section{Application to Sălăgean Derivative Operator}

Theorem 5.1. Let $0 \neq \alpha \in \mathbb{C}$ and $\Re\left\{\frac{1}{\alpha}\right\}>0$. Let $q$ be convex univalent in $\Delta$ with $q(0)=1$. Let

$$
\psi_{6}:=2 \alpha \frac{\mathscr{D}^{m} f}{z}+\frac{\left\{\mathscr{D}^{m} f\right\}^{2}}{z^{m+1} f}\left[1-\alpha-\frac{\alpha \mathscr{D}^{m+2} f}{\mathscr{D}^{m+1} f}\right]
$$

and $\chi_{6}:=q+\alpha z q^{\prime}$. Let $f \in \mathscr{A}$ and $\frac{\left\{\mathscr{D}^{m} f\right\}^{2}}{z^{m+1} f} \in \mathscr{H}[1,1] \cap \mathscr{Q}$ and $\psi_{6}$ is univalent in $\Delta$.

(i) If $\psi_{6} \prec \chi_{6}$ then

$$
\frac{\left\{\mathscr{D}^{m} f\right\}^{2}}{z \mathscr{D}^{m+1} f}<q
$$

where $q$ is the best dominant.

(ii) If $\chi_{6} \prec \psi_{6}$ then

$$
q \prec \frac{\left\{\mathscr{D}^{m} f\right\}^{2}}{z \mathscr{D}^{m+1} f}
$$

where $q$ is the best subordinant. 
Proof. Define the function $p$ by

$$
p:=\frac{\left\{\mathscr{D}^{m} f\right\}^{2}}{z^{m+1} f} .
$$

A simple computation using (5.1) shows that

$$
\frac{z p^{\prime}}{p}=\frac{2 z\left(\mathscr{D}^{m} f\right)^{\prime}}{\mathscr{D}^{m} f}-1-\frac{z\left(\mathscr{D}^{m+1} f\right)^{\prime}}{\mathscr{D}^{m+1} f} .
$$

Using the identity

$$
z\left(\mathscr{D}^{m} f\right)^{\prime}=\mathscr{D}^{m+1} f,
$$

in (5.2) we obtain

$$
\frac{z p^{\prime}}{p}=\frac{2 \mathscr{D}^{m+1} f}{\mathscr{D}^{m} f}-1-\frac{\mathscr{D}^{m+2} f}{\mathscr{D}^{m+1} f}
$$

Note that

$$
\begin{aligned}
\psi_{6} & :=2 \alpha \frac{\mathscr{D}^{m} f}{z}+\frac{\left\{\mathscr{D}^{m} f\right\}^{2}}{z \mathscr{D}^{m+1} f}\left[1-\alpha-\frac{\alpha \mathscr{D}^{m+2} f}{\mathscr{D}^{m+1} f}\right] \\
& =p+\alpha z p^{\prime}
\end{aligned}
$$

and this can be written as (2.1) when $\theta(w):=w$ and $\phi(w):=\alpha$. Now the result (i) follows as an application of Theorem 2.1. A similar exercise using Theorem (2.2) will give the result(ii).

Theorem 5.2. Let $\alpha, \beta$ and $\gamma$ be complex numbers and $\gamma \neq 0$. Let $q$ be a convex univalent in $\Delta$ with $q(0)=1$ and $\frac{\gamma z q^{\prime}}{q}$ is starlike univalent in $\Delta$. Let

$$
\psi_{7}:=\alpha-\gamma+\frac{2 \gamma \mathscr{D}^{m-1} f}{\mathscr{D}^{m} f}-\frac{\mathscr{D}^{m+2} f}{\mathscr{D}^{m+1} f}+\frac{\beta\left\{\mathscr{D}^{m} f\right\}^{2}}{z \mathscr{D}^{m+1} f}
$$

and $\chi_{7}:=\alpha+\beta q+\frac{\gamma z q^{\prime}}{q}$. Let $f \in \mathscr{A}$ and $\frac{\left\{\mathscr{D}^{m} f\right\}^{2}}{z \mathscr{D}^{m+1} f} \in \mathscr{H}[1,1] \cap \mathscr{Q}$ and $\psi_{7}$ is univalent in $\Delta$.

(i) If q satisfies (3.4), then

$$
\psi_{7} \prec \chi_{7} \Rightarrow \frac{\left\{\mathscr{D}^{m} f\right\}^{2}}{z_{D^{m+1} f}}<q
$$

where $q$ is the best dominant.

(ii) If $q$ satisfies (3.5), then

$$
\chi_{7} \prec \psi_{7} \Rightarrow q \prec \frac{\left\{\mathscr{D}^{m} f\right\}^{2}}{z \mathscr{D}^{m+1} f}
$$

where $q$ is the best subordinant.

Proof. The proof follows as an application of Theorem 2.1 and Theorem 2.2 with $\theta(w)=$ $\alpha+\beta w$ and $\phi(w)=\frac{\gamma}{w}$.

Sandwich results for the Theorems 3.3-5.2 can be obtained by a similar exercise as we have obtained the sandwich result(Theorem 3.2) of Theorem 3.1, however we omit the details of the proof. 


\section{References}

[1] T. Bulboacă, A Class of superordination preserving integral operators, Indag. Math. (N. S.) 13(2002), 301-311.

[2] T. Bulboacă, Classes of first-order diferential superordinations, Demonstratio. Math. 35(2002), 287292.

[3] S. S. Miller and P. T. Mocanu, Subordinants of differential superordinations, Complex Var. Theory Appl. 48(2003), 815-826.

[4] S. S. Miller and P. T. Mocanu, Differential subordinations: Theory and Applications, Pure and Applied Mathematics, 225, Marcel Dekker, New York, (2000).

[5] B. C. Carlson and D. B. Shaffer, Starlike and prestarlike hypergeometric functions, SIAM J. Math. Anal. 15(1984), 737-745.

[6] S. Ruscheweyh, New criteria for univalent functions, Proc. Amer. Math. Soc. 49(1975), 109-115.

[7] G. S. Sălăgean, Subclasses of univalent functions, Lecture Notes in Math., Springer-Verlag, 1013(1983), 362-372.

Department of Mathematics, Government Arts College, Paramakudi 623 707, India.

E-mail: natarajan.marikkannan@gmail.com

Department of Mathematics, Alagappa University, Karaikudi, India.

E-mail: ganesamoorthyc@yahoo.com 